\title{
POINTWISE MULTIPLIERS OF WEIGHTED BMO SPACES
}

\author{
STEVEN BLOOM
}

(Communicated by J. Marshall Ash)

\begin{abstract}
An atomic decomposition is obtained for dyadic weighted $H^{1}$ spaces with dual weighted dyadic BMO. All multipliers of dyadic weighted BMO and weighted BMO are characterized. As an application, the behavior of "logarithms" of BMO matrices are analyzed for weighted norm inequalities.
\end{abstract}

\section{BMO MULTIPLIERS}

On R, let

$$
\mathscr{D}_{t}=\left\{\text { intervals of the form }\left[t+k 2^{n}, t+(k+1) 2^{n}\right]: k, n \in Z\right\}
$$

so these are $t$-translations of the dyadic intervals $\mathscr{D}_{0}$. Thus if $I$ and $J$ in $\mathscr{D}_{t}$, either $I$ and $J$ are disjoint or one contains the other. The maximal operator with respect to $\mathscr{D}_{t}$ is

$$
M_{t}^{*} f(x)=\sup \left\{|I(f)|: x \in I, I \in \mathscr{D}_{t}\right\}
$$

where $I(f)=\left(1 /|I| \int f(x) d x\right.$. This dyadic maximal operator is related to the Hardy-Littlewood maximal operator

$$
f^{*}(x)=\sup _{x \in I} I(|f|) \text {. }
$$

On occasion we will restrict $x$ and $I$ to $\left[0,2^{n}\right]$. Then we will write this as $f^{*, n}(x)$.

A $w$-atom is a function supported in an interval $I$ with $\int a=0$ and $\|a\|_{\infty} \leq$ $1 / w(I)$, where $w(I)$ is the $w$-measure of $I$. Throughout $w$ will be a doubling measure, that is a non-negative function satisfying the property:

$$
w(J)=\int_{J} w(x) d x \leq C w(I) \quad \text { whenever } I \subset J \text { and }|J| \leq 2|I| .
$$

If $w \in L^{1}(\mathbb{R})$, we also include the constant atom $a \equiv 1$ among the $w$-atoms.

$f \in H_{u}^{1}$ if there exists $w$-atoms $\left\{a_{n}\right\}$ and a sequence $\left\{\lambda_{n}\right\} \in l^{1}(R)$ such that $f=\sum \lambda_{n} a_{n}$, and

$$
\|f\|_{w}=\inf \left\{\sum\left|\lambda_{n}\right|: f=\sum \lambda_{n} a_{n}\right\}
$$

Received by the editors January 21, 1988 and, in revised form, September 13, 1988.

1980 Mathematics Subject Classification (1985 Revision). Primary 42B25; Secondary 42B20, $42 \mathrm{~A} 50$. 
We say that $f \in H_{w, t}^{1}$ if $\|f\|_{w, t}=\int M_{t}^{*} f(x) w(x) d x<\infty$. A $w$, t-atom is a $w$-atom associated to an interval $I \in \mathscr{D}_{t}$.

Theorem 1.1. $f \in H_{w, t}^{1}$ if and only if there exist $w, t$-atoms $a_{n}$ and $\left\{\lambda_{n}\right\} \in$ $l^{l}(R)$ with $f=\sum \lambda_{n} a_{n}$. Moreover, the two norms $\|f\|_{w, t}$ and $\inf _{f=\sum \lambda_{n} a_{n}} \sum\left|\lambda_{n}\right|$ are equivalent.

This is quite standard (see, for instance [2]).

A function $b \in \mathrm{BMO}_{w, t}$ if

$$
\sup \left\{\frac{1}{w(I)} \int_{I}|b-I(b)|: I \in \mathscr{D}_{t}\right\}<\infty .
$$

This supremum is denoted by $\|b\|_{\mathrm{BMO}_{u, t}}$, and is in fact a norm on $\mathrm{BMO}_{w, t}$ modulo constants. Similarly $\mathrm{BMO}_{w}$ is described by taking this supremum over arbitrary intervals. Another standard result (see [5])is

Theorem 1.2. The dual space of $H_{w, t}^{1},\left(H_{w, t}^{1}\right)^{*}$ is $\mathrm{BMO}_{w, t}$, and $\left(H_{w}^{1}\right)^{*}=$ $\mathrm{BMO}_{w}$.

We want to characterize functions $\varphi$ such that $\varphi\left(\mathrm{BMO}_{w}\right) \subset \mathrm{BMO}_{w}$. Specifically, let $M_{\varphi}$ be the multiplication operator

$$
M_{\varphi} f(x)=\varphi(x) f(x) .
$$

For what functions $\varphi$ is $M_{\varphi}$ a bounded operator on $\mathrm{BMO}_{w}$ ? In the unweighted case on the torus $T$, Stegenga [7] solved this problem by using $H^{1}$ - BMO duality and Toeplitz theory. It should be easier and more natural to shift the problem to atoms and use some sort of $L^{1}$ check condition like Theorem 1.1. Admittedly, Theorem 1.1 only applies to the dyadic case, but the general case follows quite easily from the dyadic one. That's the approach we take to the weighted problem.

Theorem 1.3. Let $w$ as usual be a doubling measure. Then $M_{\varphi}$ is bounded on $\mathrm{BMO}_{w, t}$ if and only if $M_{\varphi}$ is bounded on $H_{w, t}^{1}$. Likewise $M_{\varphi}$ is bounded on $\mathrm{BMO}_{u}$, if and only if $M_{\varphi}$ is bounded on $H_{w}^{1}$.

Proof. Suppose $M_{\varphi}$ is bounded on $\mathrm{BMO}_{w}$. For $f \in H_{u}^{1}$,

$$
\begin{aligned}
\left\|M_{\varphi}\right\|_{w} & \leq C \sup \left\{\left|\int\left(M_{\varphi} f\right) b\right|:\|b\|_{\mathrm{BMO}_{w}} \leq 1\right\} \\
& =C \sup \left\{\left|\int f\left(M_{\varphi} b\right)\right|:\|b\|_{\mathrm{BMO}_{u}} \leq 1\right\} \\
& \leq C\left\|M_{\varphi}\right\| \cdot\|f\|_{u},
\end{aligned}
$$

so $M_{\varphi}$ is bounded on $H_{w}^{1}$. Conversely, if $b \in \mathrm{BMO}_{w}$,

$$
\left\|M_{\varphi} b\right\|_{\mathrm{BMO}_{u},} \leq C \sup \left\{\left|\int f \varphi b\right|:\|f\|_{w} \leq 1\right\} \leq C\left\|M_{\varphi}\right\| \cdot\|b\|_{\mathrm{BMO}_{u}} .
$$


The argument is identical for $H_{w, t}^{1}$ and $\mathrm{BMO}_{w, t}$.

For an interval $I$, we let $x_{I}$ denote the center of $I$ :

Theorem 1.4. Let $w$ be a doubling measure. Then $M_{\varphi}$ is bounded on $\mathrm{BMO}_{w, t}$ if and only if

(a) $\varphi \in L^{\infty}$, and

(b) $\int_{I}|\varphi-I(\varphi)| \leq C w(I)\left[\int_{x \notin I} \frac{w(x) d x}{\left|x-x_{I}\right|}\right]^{-1}$, for all $I \in \mathscr{D}_{t}$.

Proof. We take $t=0$. Fix $I \in \mathscr{D}_{0}$, with left and right halves $L$ and $R$. Put

$$
a(x)=\frac{1}{w(I)}\left[\chi_{R}-\chi_{L}\right](x) \text {. }
$$

So $a$ is a $w, 0$-atom. Set

$$
b(x)=w(x) \operatorname{sgn}(a \varphi)(x) .
$$

For any interval $J$,

$$
\frac{1}{w(J)} \int_{J}|b-J(b)| \leq \frac{2}{w(J)} \int_{J}|b|=2
$$

so $b \in \mathrm{BMO}_{w} \subset \mathrm{BMO}_{w, 0}$ and hence,

$$
\left|\int a \varphi b\right| \leq C \text {. }
$$

But $a \varphi b=|\varphi| w \mathscr{X}_{I} \frac{1}{w(I)}$, so that

$$
\frac{1}{w(I)} \int_{I}|\varphi| w \leq C .
$$

Since this holds for all dyadic $I$, if $x$ is a Lebesgue point, $|\varphi(x)| \leq 2 C$ and so $\varphi \in L^{\infty}$.

For (b), fix $I \in \mathscr{D}_{0}$. Let $g(x)=\operatorname{sgn}[\varphi(x)-I(\varphi)]$ and let $a=(1 / 2 w(L))$ $[g-I(g)] \chi_{I}$. So $a$ is a $w, 0$ atom. For $x \notin I$,

$$
\begin{aligned}
M_{0}^{*}(a \varphi)(x) & =\sup \left\{|J(a \varphi)|: x \in J, J \in \mathscr{D}_{0}, I \subset J\right\} \\
& =\sup \left\{\frac{1}{|J|}\left|\int_{I} a \varphi\right|: x \in J, J \in \mathscr{D}_{0}, I \subset J\right\} \\
& \geq \frac{1}{4\left|x-x_{I}\right|}\left|\int_{I} a \varphi\right| .
\end{aligned}
$$

But,

$$
\begin{aligned}
\int_{I} a \varphi & =\frac{1}{2 w(I)}\left[\int_{I} \varphi g-\int_{I} \varphi I(g)\right] \\
& =\frac{1}{2 w(I)}\left[\int_{I} \varphi g-|I| I(g) I(\varphi)\right] \\
& =\frac{1}{2 w(I)}\left[\int_{I} g(\varphi-I(\varphi))\right] \\
& =\frac{1}{2 w(I)} \int_{I}|\varphi-I(\varphi)|,
\end{aligned}
$$


so that

$$
M_{0}^{*}\left({ }_{a}\right) \geq \frac{1}{8 w(I)\left|x-x_{I}\right|} \int_{I}|\varphi-I(\varphi)| .
$$

Since $M_{0}^{*}(a \varphi) \in L^{1}(w)$, we have

$$
\frac{1}{w(I)} \int_{I}|\varphi-I(\varphi)| \int_{x \notin I} \frac{w(x)}{\mid x-x_{I}} \mid \leq C,
$$

which is (b).

Conversely, let $a$ be a $w, 0$ atom supported in a dyadic interval $I$. For $x \notin I$,

$$
\begin{aligned}
M_{0}^{*}(a \varphi)(x) & =\sup \left\{\frac{1}{|J|}\left|\int_{I} a \varphi\right|: x \in J, J \in \mathscr{D}_{0}, I \subset J\right\} \\
& \leq \frac{1}{\left|x-x_{I}\right|}\left|\int_{I} a \varphi\right| \\
& =\frac{1}{\left|x-x_{I}\right|}\left|\int_{I}[\varphi-I(\varphi)] a\right| \\
& \leq \frac{1}{w(I)\left|x-x_{I}\right|} \int_{I}|\varphi-I(\varphi)|
\end{aligned}
$$

so $\int_{x \notin I} M_{0}^{*}(a \varphi)(x) w(x) d x \leq C$ by $(\mathbf{b})$.

For $x \in I$,

$$
M_{0}^{*}(a \varphi)(x) \leq(a \varphi)^{*}(x) \leq\|a \varphi\|_{\infty} \leq\|\varphi\|_{\infty} / w(I),
$$

and

$$
\int_{I} M_{0}^{*}(a \varphi) w \leq\|\varphi\|_{\infty} .
$$

So $\|a \varphi\|_{w, 0} \leq C$ and hence, by Theorem 1.3, $\varphi$ multiplies $\mathrm{BMO}_{w, 0}$.

One consequence of Theorem 1.4 is that, unless $w(x) /(|x|+1) \in L^{1}$, the only multipliers of $\mathrm{BMO}_{w, t}$ are the constant functions. For the next result, we will restrict our attention to such weights.

Theorem 1.5. Let $w$ be a doubling measure, with $w(x) /(|x|+1) \in L^{1}(\mathbf{R})$. Then $M_{\varphi}$ is bounded on $\mathrm{BMO}_{u}$, if and only if

(a) $\varphi \in L^{\infty}$, and

(b) $\int_{I}|\varphi-I(\varphi)| \leq C w(I)\left[\int_{x \notin I} \frac{w(x) d x}{\mid x-x_{I}}\right]^{-1}$, for all intervals $I$.

Proof. Suppose (a) and (b) hold. Fix $I=[t, t+h]$ and $b \in \mathrm{BMO}_{w}$. Then $I \subset J=\left[t, t+2^{n}\right]$ where $2^{n} \leq 2 h . J \in \mathscr{D}_{t}$, and Theorem 4 gives $\|\varphi b\|_{\mathrm{BMO}_{w, t}} \leq$ $C\|b\|_{\mathrm{BMO}^{\prime \prime, ~},} \leq C\|b\|_{\mathrm{BMO}}$. But then,

$$
\begin{aligned}
\frac{1}{w(I)} \int_{I}|\varphi b-I(\varphi b)| & \leq \frac{C}{w(J)} \int_{J}|\varphi b-I(\varphi b)| \\
& \leq \frac{2 C}{w(J)} \int_{J}|\varphi b-J(\varphi b)| \\
& \leq 2 C\|\varphi b\|_{\mathrm{BMO}_{w, I}}
\end{aligned}
$$

so $\varphi$ multiplies $\mathrm{BMO}_{w}$. 
Conversely, if $\varphi$ multiplies $\mathrm{BMO}_{w}$, then the identical argument of the first paragraph of 1.4 shows that $\varphi \in L^{\infty}$. Fix $I=[-h, h]$ and put

$$
f(x)=\int_{|y| \geq|x|} \frac{w(y)}{|y|} d y .
$$

Fix $J=[a, a+\delta], a \geq 0$. Then

$$
J(f)-f(a+\delta)=\frac{1}{\delta} \int_{a}^{a+\delta} \int_{x}^{a+\delta} \frac{w(y)+w(-y)}{y} d y d x .
$$

We estimate $\kappa=(1 / \delta) \int_{a}^{a+\delta} \int_{x}^{a+\delta}(w(y) / y) d y d x$. When $a \geq \delta$, a simple estimate shows $\kappa \leq(\delta / a) J(w)$, while if $a<\delta$,

$$
\kappa \leq \frac{1}{\delta} \int_{0}^{2 \delta} \int_{x}^{2 \delta} \frac{w(y)}{y} d y d x=\frac{1}{\delta} \int_{0}^{2 \delta} \int_{0}^{y} \frac{w(y)}{y} d x d y=\frac{1}{\delta} \int_{0}^{2 \delta} w \leq C J(w),
$$

by doubling. A similar argument applies to any interval, proving that $f \in$ $\mathrm{BMO}_{w}\left(\mathrm{BLO}_{w}\right.$, in fact $)$. The calculation above also shows that

$$
I(f)=I(w)+\int_{x \notin I} \frac{w(x)}{|x|} d x .
$$

Both $f$ and $f-I(f)$ are in the dual of $H_{w}^{1}$. Let $g=\operatorname{sgn}(\varphi-I(\varphi))$ and put $a=(1 / 2 w(I))(g-I(g))$. So $a$ is a $w$-atom, and $\int a \varphi=(1 / 2 w(I)) \int_{I}|\varphi-I(\varphi)|$. Since $\varphi$ multiplies $H_{w}^{1},\left|\int a \varphi f\right|$ and $\left|\int a \varphi(f-I(f))\right|$ are bounded, and so their difference must be bounded. This difference is

$$
|I(f)| \frac{1}{2 w(I)} \int_{I}|\varphi-I(\varphi)| \text {. }
$$

The boundedness of this, together with (1) and $\varphi \in L^{\infty}$ gives (b).

Now suppose $w(x) /(1+|x|) \notin L^{1}$, as is the case with unweighted BMO. A weight $w \in B_{2}$ provided

$$
\frac{|I|^{2}}{w(I)} \int_{x \notin I} \frac{w(x) d x}{\left|x-x_{I}\right|^{2}} \leq C
$$

for all intervals $I$, and $w \in A_{p}$ if $I(w) I\left(w^{-1 / p-1}\right)^{p-1} \leq C$ for all intervals $I$, and $p>1$. Let $H$ be the Hilbert transform, $H f(\dot{x})=p \cdot v \cdot \int_{\mathbf{R}} \frac{f(y)}{x-y} d y$. The Hilbert transform is bounded on $L^{p}(w)$ if and only if $w \in A_{p}$ [6]. In the unweighted case, $H^{1}$ can be characterized by all functions for which $f$ and $H f \in L^{1}(\mathbb{R})$. This characterization extends to weighted $H_{w}^{1}$, so long as $w \in A_{2}$, and $H f \in L^{1}(w)$ for all $f \in H_{w}^{1}$ if and only if $w \in B_{2} \cap A_{p}$, for some $p>1[2]$. We consider such weighted spaces: 
Theorem 1.6. Suppose $w \in B_{2} \cap A_{p}$, for some $1<p$, and suppose $w(x) /(1+$ $|x|) \notin L^{1}(\mathbf{R})$. Then the only multipliers of $\mathrm{BMO}_{w}$ are the constants.

Proof. Suppose $\varphi$ multiplies $\mathrm{BMO}_{w}$ and so also $H_{w}^{1}$. As before, $\varphi$ must be in $L^{\infty}$. Let $I=\left[\frac{1}{2}, \frac{1}{2}\right]$ and let $a$ be a $w$-atom supported in $I$. For $x \geq 1$,

$$
H(a \varphi)(x)=\int_{-1 / 2}^{1 / 2} \frac{a(y)}{x-y}[\varphi(y)-I(\varphi)] d y+I(\varphi) H a(x) .
$$

Since $\varphi$ is bounded, $I(\varphi) H a \in L^{1}(w)$, and so must be

$$
b(x)=\int_{I} \frac{a(y)}{x-y}[\varphi(y)-I(\varphi)] .
$$

In particular, if we take $a=\operatorname{sgn}[\varphi-I(\varphi)] \chi_{I} / 2 w(I)\|\varphi\|_{\infty}$, then

$$
b(x)=\frac{1}{x} \frac{1}{2 w(I)\|\varphi\|_{\infty}} \int_{I}|\varphi-I(\varphi)| \in L^{1}(w) \quad \text { on }[1, \infty),
$$

and similarly on $(-\infty,-1]$ But that's impossible, unless $\int|\varphi-I(\varphi)|$ is zero, in other words, unless $\varphi$ is constant a.e. on $I$. The same argument applies to any other interval.

Thus far, we have characterized the functions $\varphi$ for which

$$
\left\|M_{\varphi} b\right\|_{\mathrm{BMO}_{w}} \leq C\|b\|_{\mathrm{BMO}_{w}} .
$$

But there certainly could exist, in theory anyway, multipliers that weren't so nicely controlled. The closed graph theorem rules out any such multipliers, so that we have:

Theorem 1.7. Let $w$ be a doubling measure, with $w(x) /(1+|x|) \in L^{1}$. Then $M_{\varphi}: \mathrm{BMO}_{w} \rightarrow \mathrm{BMO}_{w}$ if and only if

(a) $\varphi \in L^{\infty}$, and

(b) $\int_{I}|\varphi-I(\varphi)| \leq C w(I)\left[\int_{x \notin I} \frac{w(x) d x}{\left|x-x_{I}\right|}\right]^{-1}$, for all intervals $I$.

Proof. We will norm $\mathrm{BMO}_{w}$ with the constants admitted by the norm

$$
\|b\|=\left|\frac{1}{2} \int_{-1}^{+1} b(x) d x\right|+\sup _{I} \frac{1}{w(I)} \int_{I}|b-I(b)| .
$$

It is easy to see that this is a norm, providing we identify functions that agree almost everywhere. Now suppose that $\left\{f_{n}\right\}$ is a Cauchy sequence in $\mathbf{B M O}_{w}$, with this norm. Let $I_{m}=\left[2^{m}, 2^{m+1}\right]$. Then

$$
\int_{I_{m}}\left|f_{n}-I_{m}\left(f_{n}\right)-f_{k}+I_{m}\left(f_{k}\right)\right| \leq w\left(I_{m}\right)\left\|f_{n}-f_{k}\right\| \rightarrow 0 \quad \text { as } n, k \rightarrow \infty .
$$

So $\left\{f_{n}-I_{m}\left(f_{n}\right)\right\}$ is a Cauchy sequence in $L^{1}\left(I_{m}\right)$ and has a limit point $g \in$ $L^{1}\left(I_{m}\right)$. 
Now for $f \in \mathrm{BMO}_{w}$, put $J=I_{m-1} \cup I_{m}$. Then

$$
\begin{aligned}
\left|I_{m}(f)-I_{m-1}(f)\right| & \leq \frac{1}{2^{m}} \int_{I_{m}}|f-J(f)|+\frac{1}{2^{m-1}} \int_{I_{m-1}}|f-J(f)| \\
& \leq \frac{3}{2} J(w)\|f\| \\
& \leq C w\left(I_{m}\right) 2^{-m}\|f\|, \quad \text { by doubling. }
\end{aligned}
$$

So if $I=[-1,1]$,

$$
\left|I_{m}(f)-I(f)\right| \leq C w\left(I_{m}\right)\|f\|,
$$

or $\left|I_{m}(f)\right| \leq\left[C w\left(I_{m}\right)+1\right]\|f\|$. In particular, $\left\{I_{m}\left(f_{n}\right)\right\}$ is Cauchy in $\mathbb{R}$ and so converges to a number $\alpha$. So if $f=g+\alpha, f_{n} \rightarrow f$ in $L^{1}\left(I_{m}\right)$.

In this way, we can find an $f$ such that on each $I_{m}$ (or $-I_{m}$ or $I$ ), $f_{n} \rightarrow f$ in $L^{1}$. Now fix an interval $I$. Since $I \subset\left[-2^{m}, 2^{m}\right]$ for some $m$, and since $f_{n} \rightarrow f$ in $L^{1}\left(\left[-2^{m}, 2^{m}\right]\right), f_{n} \rightarrow f$ in $L^{1}(I)$, and so some subsequence $\left\{f_{n_{k}}\right\}$ converges to $f$ a.e. By Fatou's Lemma,

$$
\begin{aligned}
\frac{1}{w(I)} \int_{I}\left|f-I(f)-f_{n}+I\left(f_{n}\right)\right| & =\frac{1}{w(I)} \int_{I} \lim _{k \rightarrow \infty} \inf \left|\left(f_{n_{k}}-f_{n}\right)-I\left(f_{n_{k}}-f_{n}\right)\right| \\
& \leq \liminf _{k \rightarrow \infty} \frac{1}{w(I)} \int_{I}\left|\left(f_{n_{k}}-f_{n}\right)-I\left(f_{n_{k}}-f_{n}\right)\right| \\
& \leq \liminf _{k \rightarrow \infty}\left\|f_{n_{k}}-f_{n}\right\| \\
& \rightarrow 0 \text { as } n \rightarrow \infty .
\end{aligned}
$$

So $f_{n} \rightarrow f$ in $\mathrm{BMO}_{w}$, and thus $\mathrm{BMO}_{w}$ is a Banach space, in the norm $\|\cdot\|$. Likewise $\mathrm{BMO}_{u}$ modulo constants is a Banach space in the norm $\|\cdot\|_{\mathrm{BMO}_{m}}$.

Now suppose $M_{\varphi}: \mathrm{BMO}_{w} \rightarrow \mathrm{BMO}_{w}$. We will apply the closed graph theorem. Assume $f_{n} \rightarrow f$ in $\mathrm{BMO}_{w} /$ const , and $M_{\varphi} f_{n} \rightarrow g$ also in $\mathrm{BMO}_{w} /$ const . Restrict attention to the interval $\left[-2^{m}, 2^{m}\right]$, and let $I=[-1,1]$, as above. Choose representatives of $f_{n}$ for which $I\left(f_{n}\right)=0$ for each $n$. Then $f_{n} \rightarrow f$ in $L^{1}\left(\left[-2^{m}, 2^{m}\right]\right)$, and $I(f)=0$. By going to a subsequence, we can assume with no loss in generality that $f_{n} \rightarrow f$ a.e. in $\left[-2^{m}, 2^{m}\right]$. Hence, for almost all such $x$,

$$
M_{\varphi} f(x)=\varphi(x) f(x)=\varphi(x) \lim _{n \rightarrow \infty} f_{n}(x)=\lim _{n \rightarrow \infty}\left(M_{\varphi} f_{n}\right)(x) .
$$

In particular, this latter limit exists. Now $M_{\varphi} f_{n}-I\left(M_{\varphi} f_{n}\right) \rightarrow h$ in $L^{1}$, for some representative $h$ of the coset $g$, and so some subsequence of this converges a.e., that it

$$
M_{\varphi} f_{n_{k}}-I\left(M_{\varphi} f_{n_{k}}\right) \rightarrow h \quad \text { a.e. and } \quad I\left(M_{\varphi} f_{n_{k}}\right) \rightarrow \alpha .
$$

Hence,

$$
\lim _{k \rightarrow \infty}\left(M_{\varphi} f_{n_{k}}\right)(x)=h(x)+\alpha \text { a.e. }
$$

Thus, $M_{\varphi} f=h+\alpha=g$ in $\mathrm{BMO}_{w} /$ const. 
So $M_{\varphi}$ is a continuous linear operator on $\mathrm{BMO}_{w} /$ const, and combining with Theorem 1.5 gives the result.

\section{Applications}

Nowhere in the arguments of the previous section was any one-dimensional property used. With no more than trivial changes, we have

Corollary 2.1. $\varphi$ multiplies $\mathbf{B M O}_{w, t}\left(\mathbf{R}^{n}\right)$ or $\mathrm{BMO}_{w}\left(\mathbb{R}^{n}\right)$ if and only if

(a) $\varphi \in L^{\infty}$, and

(b) $\sup \frac{1}{w(Q)} \int_{x \notin Q} \frac{w(x)}{\left|x-x_{Q}\right|} n d x \int_{Q}|\varphi-Q(\varphi)| \leq C$,

where this supremum is taken over all t-translates of dyadic cubes for $\mathrm{BMO}_{w, t}$ and over all cubes for $\mathrm{BMO}_{w}$. Here $w$ is assumed to be doubling, and for $\mathrm{BMO}_{w}, w(x) /\left(1+|x|^{n}\right) \in L^{1}$.

On the torus $T$ in the complex plane, let $\mathscr{D}_{t}$ be the dyadic intervals in $T$ treating $T$ as $[t, t+2 \pi]$. And $w, t$-atoms are defined as usual, except that $a \equiv 1$ is a $w, t$-atom also. Everything carries through to this setting (Theorem 1.2 is easier).

Corollary 2.2. $\varphi$ multiplies $\mathrm{BMO}_{u, t}(T)$ or $\mathrm{BMO}_{u}(T)$ if and only if

(a) $\varphi \in L^{\infty}$, and

(b) $\sup \frac{1}{w(I)} \int_{x \notin I} \frac{w(x)}{\left|x-x_{I}\right|} d x \int_{I}|\varphi-I(\varphi)| \leq C$,

where the supremum is taken over these t-dyadic intervals for $\mathrm{BMO}_{w, t}$ or over all intervals for $\mathrm{BMO}_{w}$. Here, as usual, $w$ is doubling.

When

$$
w \equiv 1, \int_{x \notin I} \frac{w(x)}{\left|x-x_{I}\right|} d x \cong \log \frac{1}{|I|},
$$

and we get Stegenga's Theorem: $\varphi$ multiplies $\operatorname{BMO}(T)$ if and only if $\varphi \in L^{\infty}$ and

$$
\frac{1}{|I|} \log \frac{1}{|I|} \int_{I}|\varphi-I(\varphi)| \leq C .
$$

For noncompact spaces like $\mathbb{R}^{n},\left(1 /\left|x-x_{Q}\right|\right) \chi_{\mathbf{R}} n_{\sim Q} \notin L^{1}$, which means that only constants multiply $\operatorname{BMO}\left(\mathbf{R}^{n}\right)$. This is disappointing, although, as Ron Kerman pointed out, it is a powerful reason for using weights.

A harder problem is to determine the multipliers of $\operatorname{BMO}(I)$ where $I$ is a bounded interval in $R$ or cube in $\mathbb{R}^{n}$. The methods developed in $\S 1$ can be used effectively for such problems, and yield the obvious analogs of Stegenga's theorem. We leave the details to the reader.

As a final application, we will look at a matrix analog of $A_{p}$ weights. Much of the following material appeared in [3], and for more details, the interested reader is referred there. 
Let $H$ denote the conjugate operator, or Hilbert transform, on the unit circle $T$. The commutator $\left[H, M_{b}\right] f=H(b f)-b(H f)$ is bounded on $L^{2}(T)$ if and only if $b \in$ BMO [4]. Let $L_{n}^{2}(T)$ denote the space of $n$-vectors with

$$
\|f\|_{2}=\left[\frac{1}{2 \pi} \int_{T}<f(t), f(t)>d t\right]^{1 / 2}<\infty .
$$

We say an $n \times n$ matrix $B \in$ BMO if each entry $b_{i j} \in$ BMO.

Theorem 2.3. $\left[H, M_{b}\right]$ is bounded on $L_{n}^{2}(T)$ if and only if $B \in \mathrm{BMO}$. Moreover,

$$
\max _{i, j}\left\|b_{i j}\right\|_{\mathrm{BMO}}
$$

is equivalent as a norm to the operator norm of $\left[H, M_{b}\right]$.

Proof. By writing $f$ as $\sum f_{i} e_{i}$ where $\left\{e_{i}\right\}$ is the standard basis and $f_{i}$ are scalar valued, it is easy to see that $\left[H, M_{b}\right]$ is bounded on $L_{n}^{2}(T)$ if and only if each $\left[H, M_{b}\right]$ is bounded on $L^{2}(T)$, and the theorem reduces to the onedimensional theory.

Let $W$ be a matrix-valued function on $T$ with $W(t)$ positive-definite and symmetric. We have an inner product

$$
(f, g)_{W}=\frac{1}{2 \pi} \int_{T}<W(x) f(x), g(x)>d x
$$

which induces a Hilbert space of vector-valued functions $L^{2}(W)$. The matrix analog of $A_{2}$ is:

$$
W \in \mathscr{C}_{2} \quad \text { if }\|H f\|_{W} \leq C\|f\|_{W} \quad \text { for all } f \in L^{2}(W) .
$$

For diagonal matrices, $W \in \mathscr{C}_{2}$ if and only if each $w_{i i} \in A_{2}$. For a discussion of more general matrices in $\mathscr{C}_{2}$, see [1] or [3].

Theorem 2.4. If $W \in \mathscr{C}_{2}$, so is $W^{-1}$.

Proof. The adjoint of $H$ on $L^{2}(W)$ is

$$
H^{*} f=-W^{-1} H(W f) .
$$

Since $W \in \mathscr{C}_{2}, H^{*}$ is bounded on $L^{2}(W)$, and so

$$
\begin{aligned}
\|H f\|_{W^{-1}}^{2} & =\frac{1}{2 \pi} \int\left\langle W^{-1} H f, H f\right\rangle \\
& =\frac{1}{2 \pi} \int\left\langle W W^{-1} H f, W^{-1} H f\right\rangle \\
& =\left\|W^{-1} H f\right\|_{W}^{2} \\
& =\left\|H^{*}\left(W^{-1} f\right)\right\|_{W}^{2} \\
& \leq C\left\|W^{-1} f\right\|_{W}^{2} \\
& =C\|f\|_{W-1}^{2} \cdot \square
\end{aligned}
$$


Since $W$ is positive-definite, it has a logarithm $B$. Let

$$
T_{z}=e^{z B} H e^{-z B} \text {. }
$$

Since $W$ and $W^{-1} \in \mathscr{C}_{2}, T_{ \pm 1 / 2}$ is bounded on $L_{n}^{2}(T)$, and complex interpolation gives $T_{z}$ bounded for $|z| \leq \frac{1}{2}$. By Cauchy's Theorem,

$$
T_{z}=\frac{1}{2 \pi i} \int_{|\zeta|=1 / 2} \frac{T_{\zeta}}{\zeta-z} d \zeta
$$

and $\left.(d / d z) T_{z}\right|_{z=0}$ is bounded on $L_{n}^{2}(T)$. But this derivative is $\left[H, M_{B}\right]$. So we have a matrix analog of the familiar one-dimensional result:

Theorem 2.5. If $W \in \mathscr{C}_{2}$ then $\log W \in \mathrm{BMO}$.

In one dimension, if $b \in \mathrm{BMO}$, then $e^{t b} \in A_{2}$ for $t$ sufficiently small. So 2.5 more or less characterizes $A_{2}$. It is surprising that the matrix analog fails.

Theorem 2.6. There exist symmetric $B \in \mathrm{BMO}$ for which $e^{t B} \notin \mathscr{C}_{2}$ for any $t \neq 0$.

Proof. Let $v \in L^{\infty}$ be such that $v$ does not multiply BMO. Now BMO $=$ $L^{\infty}+H L^{\infty}$. Since $v L^{\infty} \subset L^{\infty} \subset \mathrm{BMO}, v\left(H L^{\infty}\right) \nsubseteq \mathrm{BMO}$, and there exists a $u \in L^{\infty}$ with $v H u \notin \mathrm{BMO}$. For $B$ we take

$$
B=\left[\begin{array}{cc}
H u & v \\
v & 0
\end{array}\right] \text {. }
$$

Suppose that $e^{t B} \in \mathscr{C}_{2}$ for some $t \neq 0$. Then as above, $T_{z}$ would be bounded in a neighborhood of zero, and all of the derivatives of $T_{z}$ at zero would be bounded operators. These are higher-order commutators, and in one dimension, if the first commutator is bounded, they all are. We look at the second commutator,

$$
B^{2} H-2 B H B+H B^{2} .
$$

Applying this to the vector $\left(\begin{array}{l}f \\ 0\end{array}\right)$ we get

$$
\left[v H u H f-2 v H\left[H^{*} u f\right]+H[v(h u) f]\right]
$$

so the operator

$$
f \rightarrow v H u H f-2 v H(f H u)+H(v f H u)
$$

is bounded.

Now $H u+c v \in$ BMO so that $e^{s H u+t v} \in A_{2}$ for $s$ and $t$ sufficiently small, and so

$$
\left.\frac{\partial}{\partial s} \frac{\partial}{\partial t} e^{s H u+t u} H e^{-s H u-t w}\right|_{(0,0)}
$$

is bounded on $L^{2}$. This is the operator

$$
f \rightarrow v H u H f-v H(H u) f-H u H v f+H(H u) v f .
$$


Subtracting (2) and (1) gives the bounded operator

$$
f \rightarrow H u H v f-v H[(H u) f] .
$$

Using elementary properties of the conjugate operator, (3) is the operator

$$
-\left[H, M_{H u}\right] M_{v}+M_{u v}+M_{v} H\left(M_{u} H\right)+\left[H, M_{(H u) v}\right] .
$$

Since $u$ and $v \in L^{\infty}$, the first three terms in (4) are bounded operators, so we conclude that $\left[H, M_{v H u}\right]$ is bounded on $L^{2}$, and hence that $v H u \in \mathrm{BMO}$, a contradiction.

\section{REFERENCES}

1. S. Bloom, A commutator theorem and weighted BMO, Trans. Amer. Math Soc. 292 (1) (1985), 103-122.

2. __ Conjugate operator invariance of weighted BMO (to appear).

3. __ Weighted norm inequalities for vector-valued functions, $\mathrm{Ph}$. D. dissertation, Washington University, St. Louis, Missouri, 1981.

4. R. R. Coifman, R. Rochberg and G. Weiss, Factorization theorems for Hardy spaces in several variables, Ann. of Math. 103 (1976), 611-635.

5. R. R. Coifman and G. Weiss, Extensions of Hardy spaces and their use in analysis, Bull. Amer. Math. Soc. 83 (1977), 569-645.

6. R. Hunt, B. Muckenhoupt and R. Wheeden, Weighted norm inequalities for the conjugate function and the Hilbert transform, Trans. Amer. Math. Soc. 176 (1973), 227-251.

7. D. A. Stegenga, Bounded Toeplitz operators on $H^{1}$ and applications of the duality between $H^{1}$ and the functions of bounded mean oscillation, Amer. J. Math. 98 (3) (1976), 573-589.

Department of Mathematics, Siena College, Loudonville, New York 12211 